\title{
RECENT PROGRESS WITH THE DIRICHLET PROBLEM*
}

BY O. D. KELLOGG

1. The Classical Problem of Dirichlet, and its Status at the Beginning of the Twentieth Century. Let $T$ denote an open continuum in a euclidean space of $n$ dimensions, and let $t$ denote the set of its boundary points. A function, $F(p)$, defined on $t$, will be said to be continuous, if to every point, $p$, of $t$, and every $\epsilon>0$, there corresponds a $\delta>0$, such that $|F(p)-F(q)|<\epsilon$ for every point, $q$, of $t$, for which the distance $p q<\delta$. The classical problem of Dirichlet is then the following. Given $T$ and $F(p)$, to find a function continuous in $T+t$, reducing to $F(p)$ on $t$, and having in $T$ continuous partial derivatives of second order which satisfy Laplace's differential equation

$$
\nabla^{2} u=\frac{\partial^{2} u}{\partial x_{1}^{2}}+\frac{\partial^{2} u}{\partial x_{2}^{2}}+\cdots+\frac{\partial^{2} u}{\partial x_{n}^{2}}=0 .
$$

We shall limit ourselves to the cases $n \leqq 3$.

We shall be concerned rather with the existence of the solution than with its actual construction. A domain, $T$, for which a solution exists corresponding to each continuous $F(p)$, will be called a normal domain.

In one dimension, the problem is always possible, for it amounts merely to finding a straight line through two points. In two and three dimensions, the most extensive results at the close of the last century were due to Poincaré and his method of balayage. The contribution of Poin-

* An address delivered at the meeting of the Society on January 2, 1926, in New York City, by invitation of the Program Committee.

$\dagger$ Sur les équations aux dérivées partielles de la physique mathêmatique, American Journal, vol. 12 (1890), pp. 211-294, and Théorie du Potentiel Nerwtonien, Paris, 1899. Carried out in two dimensions by A. Paraf, Sur le probleme de Dirichlet, Annales DE Toulouse, vol. 6 (1892), pp. H. 1-H. 75. 
caré consisted in three elements: first, a recognition of the fact that if the problem was solvable when $F(p)$ represented the boundary values of any polynomial, it was solvable for any continuous $F(p),{ }^{*}$ secondly, a method for establishing a harmonic function (solution of Laplace's equation) corresponding to given boundary values, $F(p)$, and thirdly, a method of proof that this harmonic function assumed the assigned boundary values by means of certain dominant functions which we shall call, following Lebesgue, barriers, and consider later $(\$ 5)$. The result was that the existence of the solution of the Dirichlet problem was established for domains bounded by surfaces with finite principal curvatures at all but a finite number of ordinary conical points.

The method of integral equations, developed by Fredholm, $\dagger$ because of its powerful character, gave great impetus to the study of the Dirichlet problem. It emphasized the independence of the existence theorem of the general shape of the boundary of $T$, and proved most useful in many problems of potential theory. But it was hindered in aiding substantially the progress of the Dirichlet problem by its use of the double distribution, which carried with it the demand for a fairly smooth boundary.

The solution of a certain fundamental case of the Dirichlet problem was brought to a successful conclusion by Osgood $\ddagger$ in his proof that Green's function existed for the most general simply connected domain of the plane with more

* The reasoning was not complete, for it involved the assumption that corresponding to continuous $F(p)$ there always existed a function $F(P)$, continuous throughout $T+t$ and coinciding with $F(p)$ on $t$, a fact which had not been established at that time.

$\dagger$ Sur une nouvelle méthode pour la résolution du problème de Dirichlet, Ofversigt af Kongl. Vet. Ak. Förh., vol. 57 (1900), pp. 39 ff. Sur une classe d'équations fonctionnelles, Acta Mathematrca, vol. 27 (1903), pp. 365-390.

$\ddagger$ On the existence of the Green's function for the most general simply connected plane region, Transactions of this Society, vol. 1 (1900), pp. 310-314. 
than one boundary point. That this result implied the fact that such a domain was normal was recognized only sixteen years later, when Lichtenstein* proved that from the existence of Green's function for a domain, $T$, followed the possibility of the Dirichlet problem for any continuous $F(p)$ for that domain.

But in the mean time, Lebesgue, using the methods of the calculus of variations as refounded by Hilbert, $\uparrow$ had attained results including this.f He showed that a domain $T$ in two dimensions was normal provided no boundary point could be enclosed by a curve lying in $T$ and an arbitrarily small neighborhood of the point. In three dimensions, Zaremba $\S$ proved that $T$ was normal, provided that each boundary point was the vertex of a right circular cone lying outside of $T$ except at the vertex.

2. Limitations on the Dirichlet Problem. Thus far, writers on the subject had regarded the Dirichlet problem as always possible, and their endeavors had been to find methods of proof powerful enough to establish its possibility. A new chapter begins with the recognition of the fact that domains exist, for $n \geqq 2$, which are not normal. In 1903 , Bôcher || had proved that a singularity of a bounded harmonic function at an isolated point can only be a removable one. Zaremba pointed out that this meant that a domain whose boundary contained an isolated point could not be normal. An even more striking example was given for a

* Ueber die erste Randwertaufgabe der Potentialtheorie, Sitzungsberichte, Berliner Mathematische Gesellschaft; Archiv für Mathematik UND Physik (3), vol. 25 (1916-17), pp. 92-96.

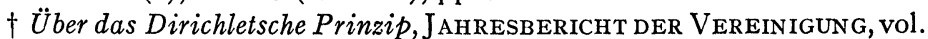
8 (1900), p. 184 et seq.

$\ddagger$ Sur le problème de Dirichlet, Rendicontr di Palermo, vol. 24 (1907), pp. 371-402.

$\S$ Sur le principe de Dirichlet, Acta Mathematica, vol. 34 (1911), pp. 293-316.

II Singular points of functions which satisfy partial differential equations of the elliptic type, this Bulletin, vol. 9 (1903), pp. 455-465.

T ACta Mathematica, vol. 34, p. 310. 
three-dimensional domain by Lebesgue*; the domain was simply connected, but its boundary contained a sharp inward projecting spine, and a certain harmonic function assuming preassigned continuous boundary values everywhere else failed to assume the given value at the point of the spine. With the emergence of facts of this character, the study of the Dirichlet problem was forced into new channels, and later researches may be fairly characterized as falling under two heads: the recognition and characterization of exceptional boundary points, and the suitable extension of the meaning of the Dirichlet problem, so that it shall admit of one, and just one, solution, no matter what the domain.

3. The Extended Function of Green. Given a domain $T$, there exists a sequence of domains $\left[T_{n}\right]$ with these properties: each is a part of all the succeeding ones, and each point of $T$ is in all of the domains of the sequence from a given one on. For instance, $T_{n}$ might be the interior points of the set of all the closed cubes of the lattice $x=a / 2^{n}, y=b / 2^{n}, z=c / 2^{n},(a, b$, and $c$ being integers) which lay in $T$. Such domains would be normal, as shown by Zaremba. If desired, these domains could also be so rounded off as to have continuous principal curvatures at all points. Also, it could be arranged that the boundary of each was also interior to those following. We shall speak of such a sequence as a set of nested domains with $T$ as limit, not necessarily requiring, however, that they be rounded off. We shall always suppose them normal.

Then, $T$ being a domain, and $Q$ any point of $T$, Green's function $G_{n}(P, Q)$ exists for each domain $T_{n}$ of a nested set as soon as $T_{n}$ contains $Q$. The sequence $\left[G_{n}(P, Q)\right]$ is monotone increasing, and, by the reasoning of Harnack, $\dagger$ approaches a limit, uniformly in any closed sub-region of $T$

* Sur des cas d'impossibilitê du problème de Dirichlet, Comptes Rendus DE LA SociétÉ DE France, 1913, p. 17 et seq. See 6, below. $\$ 39$.

$\dagger$ Grundlagen der Theorie des logarithmischen Potentials, Leipzig, 1887, 
which omits $Q$. This limit function, $G(P, Q)$, is harmonic in the sub-region. We call it Green's function for $T$, leaving open the question as to its behavior on the boundary, t.* It has these properties: $G(P, Q)-1 / P Q$, is harmonic in $T$ save for a removable singularity at $Q$; this difference is never positive; if $T$ extends to infinity, $G(P, Q)$ vanishes regularly at infinity; and $G(P, Q)=G(Q, P)$.

The points $p$ of $t$ at which $G(P, Q)$ approaches 0 are called regular boundary points. All other points of $t$ are called exceptional. If there are no exceptional boundary points, $T$ is normal. $\dagger$ But if there are exceptional points, the domain is not normal, as may be seen as follows. The Dirichlet problem for boundary values $1 / P Q$ is not possible. For, if it were, there would exist a function of Green approaching 0 at every boundary point. Such a function would dominate all the $G_{n}(P, Q)$ of our sequence, and hence their limit, $G(P, Q)$ The Green function formed in this way would then also approach 0 at every boundary point, and exceptional boundary points would then not exist.

The above definition of regular and exceptional boundary points is easily shown to be independent of the pole, $Q$, of Green's function. In fact, if $Q$ and $Q^{\prime}$ be two points of $T$, and $R$ is a closed region containing them in its interior, a certain constant multiple of $G\left(P, Q^{\prime}\right)$ dominates $G(P, Q)$ in $T-R$, so that $G(P, Q)$ approaches 0 at every boundary point at which $G\left(P, Q^{\prime}\right)$ does, and vice versa. $\ddagger$

4. Wiener's Sequence Solution of the Dirichlet Problem. Given a domain $T$, and a function, $F(p)$, continuous on its boundary, $t$, it is always possible to find a function $F(P)$, defined and continuous throughout $T+t$, and coinciding

* Kellogg, On the general solution of the classical Dirichlet problem, Proceedings of the National Academy, vol. 12 (1926), pp. 397-406. Referred to later as I.

$\dagger$ Lichtenstein, loc. cit. In three dimensions, Kellogg, loc. cit., I.

$\ddagger$ Kellogg, loc. cit., I. 
with $F(p)$ on $t .^{*}$ This fact permits the establishment of a function, harmonic in $t$, and corresponding to any continuous boundary values $F(p)$, in the same manner as that in which Harnack established Green's function. $\dagger$ Thus, given $T$ and the continuous boundary values $F(p)$, we determine any continuous extension, $F(P)$, of $F(p)$ to the points of $T$, and any nested set $\left[T_{n}\right]$ of normal domains. For each of these, we determine the function $u_{n}(P)$, harmonic in $T_{n}$, and approaching on the boundary, $t_{n}$, the values which $F(P)$ there assumes. If $T$ is normal, the sequence $\left[u_{n}(P)\right]$ converges uniformly to a function, harmonic in $T$, which assumes the boundary values $F(p) . \quad$ It is the great merit of Wiener to have established the facts that this sequence always converges, no matter what the domain $T$ or the continuous boundary values, $F(p)$, and that its limit, $u(P)$, is harmonic in $T$, and is independent of both the continuous extension of $F(p)$ to the points of $T$, and of the nested set $\left[T_{n}\right] . \S$ This function $u(P)$ also approaches the boundary values $F(p)$ at all regular boundary points. It is thus, in an extended sense, a solution of the Dirichlet problem which exists no matter what the domain $T$ or the continuous boundary values $F(p)$. We call it the sequence solution. We shall see later $(\$ 9)$ in what sense it may as yet be regarded as a final solution of the Dirichlet problem.

5. The Character of Boundary Points. Barriers. The method of balayage, the methods of the calculus of variations, and the above extended method of Harnack, all produce harmonic functions as limits of sequences corresponding to assigned boundary values, and all require sup-

* Lebesgue, Rendiconti di Palermo, vol. 24, p. 379; Carathéodory, Vorlesungen über reelle Funktionen, Leipzig, 1918, pp. 617-20.

$\dagger$ Kellogg, An example in potential theory, Proceedings of THE AMERICAN Academy, vol. 58 (1923), pp. 527-533. Referred to later as II.

$\ddagger$ Ibid.

$\S$ Certain notions in potential theory, Journal of MAThematics AND Physics, Mass. Inst. of Tech., vol. 3 (1924), pp. 24-51. A simplified treatment of these theorems will be found in the writer's paper I, cited above. 
plementary investigation of the behavior of this harmonic function on the boundary.* It was Lebesgue who first explicitly separated the two problems, and it was Lebesgue $\dagger$ who first generalized the barrier concept so effectively used by Poincaré. We shall define barrier in its simplest aspect; essentially the generality of other definitions is only apparent, as we shall see. Let $T$ be a bounded domain, and $p$ one of its boundary points. The function $U(P, p)$ is a barrier for $T$ at $p$ if it is harmonic in $T$, never less than $P p$, and if it approaches 0 at $p$. Using this concept, we may state the theorem: A necessary and sufficient condition that $T$ be normal is that there exist a barrier for $T$ at every boundary point of $T$.

We can go farther than this, however, and find in the barrier a criterion for the regularity of the individual boundary points. But it is first desirable to consider the notions of subharmonic and superharmonic functions. According to $\mathrm{F}$. Riesz, $\S$ a function $W(P)$, defined and continuous in $T$ (or upper semicontinuous-and it may even become negatively infinite at isolated points), is subharmonic when it possesses the following property: given any subdomain, $T^{\prime}$, of $T$, every function which is harmonic in $T^{\prime}$ and continuous in $T^{\prime}+t^{\prime}$ and which dominates $W(p)$ on $t^{\prime}$, does so also throughout $T^{\prime}$. Here $t^{\prime}$ denotes the boundary of $T^{\prime}$. A superharmonic function may be defined simply as the negative of a subharmonic function. A harmonic function belongs to both classes. The following are a few of the properties of subharmonic functions given by Riesz, and which are of importance for our present purposes. Let $A_{\sigma} W$ denote the arithmetic mean of the values of $W$

* Loc. cit., Rendiconti di Palermo, vol. 24.

† Sur le problème de Dirichlet, Comptes Rendus, vol. 154 (1912), p. 335 .

$\ddagger$ Kellogg, loc. cit., II.

$\S$ Ueber subharmonische Funktionen und ihre Rolle in der Funktionentheorie und in der Potentialtheorie, Acta, Franz-Joseph University, SzEGED, vol. 2 (1925), pp. 87-100. 
on the sphere $\sigma$ with center at $C$, and lying in a domain in which $W$ is continuous. Then $W$ is subharmonic in this domain if for every such sphere, $W(C) \leqq A_{\sigma} W$, and conversely. If $W$ has continuous second partial derivatives, $\nabla^{2} W \geqq 0$ is a necessary and sufficient condition that $W$ be subharmonic. Finally, if $[W]$ denote a set (which, for our purposes, may be finite) of functions subharmonic in $T$, and if $W(P)$ has at each point $P$ of $T$ the greatest of the values which the functions of the set assume at $P$, then $W(P)$ is also subharmonic.

It is now easy to show that a necessary and sufficient condition that $p$ be a regular boundary point of $T$ is that a barrier exist for $T$ at $p$. We first consider the case in which $T$ is bounded. Let us assume that a barrier $U(P, p)$ exists. Then $G(P, Q) \rightarrow 0$ as $P \rightarrow p$, and $p$ is regular. For if $\sigma$ be a sphere, of radius $a$ about $Q$, lying in $T$, and if $m$ be the minimum distance of the points of $\sigma$ from $t$, then on $\sigma$,

$$
\frac{1}{m a} U(P, p) \geqq \frac{1}{m a} P p \geqq \frac{1}{a}=\frac{1}{P Q} .
$$

Hence in $T_{n}-\sigma$,

$$
G_{n}(P, Q) \leqq \frac{1}{m a} U(P, p),
$$

and hence in $T-\sigma$,

$$
G(P, Q) \leqq \frac{1}{m a} U(P, p),
$$

so that $G(P, Q) \rightarrow 0$ as $P \rightarrow p$. On the other hand, if $p$ is regular, a barrier $U(P, p)$ exists. It is the sequence solution of the Dirichlet problem corresponding to the boundary values which $F(P)=P p$ assumes. For if we denote $P p$ by $r, \nabla^{2} F(P)=\nabla^{2} r=2 / r>0$, and $F(P)$ is subharmonic in $T$. Hence the sequence functions, $u_{n}(P)$, dominate $F(P)$, and so, therefore, does their limit, $U(P, p)$. But $p$ is a regular point, and hence at $p$ the sequence solution $U(P, p)$ approaches the assigned boundary value, 0 . 
So much for bounded domains. A simple expedient shows that if a barrier $U(P, p)$ exists for only so much of $T$ as is contained in a sphere, $\sigma$, about $p, p$ is regular, and conversely. Let $a$ denote the radius of $\sigma$. We define a continuous superharmonic function $W(P, p)$ as follows: within $\sigma, W(P, p)$ is at $P$ the smaller of the numbers $a$ and $U(P, p)$, or is their common value if equal; in the rest of $T, W(P, p)=a$. Thus the existence of a barrier at $p$ for merely a neighborhood of $p$ in $T$ implies the existence of the function $W(P, p)$ which function is superharmonic in $T$, not less than $P p$ in a neighborhood of $k$, and approaches 0 at $p$. A slight modification of the previous reasoning shows that $G(P, Q)$ must approach 0 at $p$. On the other hand, if $p$ is a regular boundary point of $T$, it is also a regular boundary point of any sub-domain $T^{\prime}$ of which it is a boundary point, since Green's function for $T$ dominates that for $T^{\prime}$. Hence $U(P, p)$ exists at $p$ for a neighborhood of $p$ in $T$, and the superharmonic function $W(P, p)$ exists in $T$.

Incidentally, the fact has emerged that the regularity of a boundary point is dependent purely on the $i m$ Kleinen character of the boundary at that point.*

The function $W(P, p)$ is a special case of the more general type of barrier defined by Lebesgue. $\dagger$ While the existence of either implies that of the other, the latter type is of ten more convenient to apply. $W(P, p)$ is said to be a barrier for $T$ at $p$ if $W(P, p)$ is continuous and superharmonic in $T$ and if it approaches 0 at $p$ and has a positive lower bound at every other boundary point. If $p$ is regular, such a function exists in the more special function established above. On the other hand, if $W(P, p)$ exists, the more special barrier $U(P, p)$ exists for as much of $T$ as lies in

* Lebesgue, Sur le principe de Dirichlet, Comptes Rendus, vol. 155 (1912), pp. 699-701. Kellogg, loc. cit., II.

$\dagger$ Conditions de régularité, conditions d'irrégularité, conditions d'impossibilité dans le problème de Dirichlet, Comptes Rendus, vol. 178 (1924), p. 349. 
a sphere of radius $R$ about $p$. For, outside a sphere $\sigma^{\prime}$ of radius $a$ about $p$, and in the part $T^{\prime}$ of $T$ within the sphere of radius $R, W(P, p)$ has a positive lower bound, $\delta$. This follows for boundary points, by definition, and for interior points because a superharmonic function can have no interior minima, since for them $W(C) \geqq A_{\sigma} W$. Hence, in $T^{\prime}-\sigma^{\prime}$,

so that

$$
W(P, p) \geqq \frac{P p-a}{R-a} \delta,
$$

$$
W^{\prime}(P, p)=\frac{R-a}{\delta} W(P, p)+a
$$

dominates $P p$ in $T^{\prime}$. But this function is continuous and superharmonic in $T^{\prime}$, and hence dominates the sequence functions $u_{n}(P)$ corresponding to $F(P)=P p$, and therefore their limit, $U(P, p)$. But $W^{\prime}(P, p) \rightarrow a$ as $P \rightarrow p$. Hence the upper limit of $U(P, p)$ at $p$ is not greater than $a$. But it is independent of $a$, which number may be any small positive quantity, and hence this upper limit is 0 . That is, $U(P, p)$ is a barrier for $T^{\prime}$ in the narrower sense.

6. Types of Regular and Exceptional Boundary Points. In the plane, a simply connected region with more than one boundary point has no exceptional points, as we have seen (p. 603). It follows from the im Kleinen character of regularity that any boundary point is regular if it belongs to a connected set of boundary points, containing other points. Hence if a plane domain is to have exceptional points, either its boundary must contain isolated points, or the domain must have infinite connectivity. As instances of possibilities, we give the following two examples. The first exhibits a domain of infinite connectivity whose boundary is a linear set of Borel measure 0 , yet all of whose points are regular.* In fact, the boundary set in question is Cantor's nowhere dense perfect set, being the points left of a closed segment after the removal of its open middle

* Kellogg, loc. cit., II. 
third, the removal of the open middle thirds of the two remaining segments, the removal of the middle thirds of the four remaining segments, and so on, ad infinitum.

The second example* is, in a way, an antithesis to the first. Here a boundary point is exceptional although it is a point of condensation of the boundary. One forms first the function

$$
U(x, y)=\sum_{0}^{\infty} \frac{1}{2^{n}} \log \left[\left(x-1 / 2^{n}\right)^{2}+y^{2}\right]^{-1 / 2},
$$

harmonic except at the points $\left(1 / 2^{n}, 0\right)$. It is not difficult to show that if $(x, y)$ is any regular point of $U(x, y)$,

$$
\lim _{n \rightarrow \infty} U\left(\frac{x}{2^{n}}, \frac{y}{2^{n}}\right)=2 \log 2,
$$

and that the locus $U(x, y)=5$ consists of closed oval curves each enclosing one and but one of the points $\left(1 / 2^{n}, 0\right)$. The domain, $T$, in question, consists of the points interior to a simple closed equipotential, $U(x, y)=-C$ ( $C$ a sufficiently large positive constant), except for the ovals $U(x, y)=5$, their interiors, and their single new limit point, $O$, the origin. A function harmonic in $T$ and approaching the boundary values $-C$ on the outer boundary, and 5 on the ovals and at $O$, does not exist. For, if it did, it would differ from $U(x, y)$ by a function harmonic in $T$, bounded, and approaching the boundary values 0 at every point but $O$. The difference would then vanish, being dominated (as well as its negative) by $-\epsilon \log \left[\left(x^{2}+y^{2}\right) / R^{2}\right]$ for every positive $\epsilon$, where $R$ is the maximum diameter of $T$. But $U(x, y)$ does not assume the required boundary value at $O$. Hence the Dirichlet problem in question has no solution, and $T$ is not normal. But as all other boundary points are regular, $O$ must be exceptional.

Thus points of condensation of the boundary may be exceptional. But a point cannot be regular unless it is a point of condensation of the boundary (see p. 620).

* The underlying idea in the construction of this example, I owe to a conversation with Professor Wiener. A somewhat similar example is given by Lebesgue, loc. cit., Rendiconti di Palermo, vol. 24. 
An isolated boundary point, and the point $O$ of the above example, are exceptional points of essentially different characters. The isolated point can be the seat of no singularities of bounded harmonic functions other than removable ones, and it therefore has no influence on the determination of a harmonic function by its boundary values. Such points may therefore be removed from the boundary without essential alteration of the Dirichlet problem. On the other hand, the point $O$ of the example given, cannot be removed without impairing the essential quality of the domain $T$ of being an open set, or else removing regular points at the same time. Bouligand* has made the distinction between the two kinds of exceptional boundary points on the basis of the generalized Green function. A set of points at which $\lim G(P, Q)>0$, (and such are all isolated points), he calls an improper set. At each point of an improper set, not only is $\lim G(P, Q)>0$, but $\lim G(P, Q)$ exists and is positive. $\dagger$

In a space of three dimensions, arcs of regular curves of finite length which are isolated from the rest of the boundary, contain only exceptional points-in fact, they are improper sets. $\ddagger$ The first example of an exceptional point which is not of the improper type was given by Lebesgue, $\S$ and it is to be noted that in distinction to the possibilities in the plane, this point belongs to the boundary of a simply connected domain. Consider the potential of a spread on a straight line segment whose density varies with the distance from one end:

$$
V=\int_{0}^{1} \frac{\xi d \xi}{P Q}, \quad P Q=\left((\xi-x)^{2}+y^{2}+z^{2}\right)^{1 / 2} .
$$

* Sur le problème de Dirichlet, Annales de la Société Polonaise, 1925, pp. 59-112.

$\dagger$ Kellogg, loc. cit., I.

¥Lebesgue, Sur les singularités des fonctions harmoniques, Comptes RENDUS, vol. 176 (1923), pp. 1097-99.

§ Loc. cit., p. 604, footnote 1. 
The integral may be evaluated, and gives

$V=\left(1-2 x+r^{2}\right)^{1 / 2}-r+x \log \frac{\left(1-2 x+r^{2}\right)^{1 / 2}+1-x}{r-x}$,

where

$$
r=\left(x^{2}+y^{2}+z^{2}\right)^{1 / 2} \text {. }
$$

This relation is equivalent to

$$
r=x+e^{\mu}, \quad \mu=\frac{1-V+\eta}{2 x},
$$

where $\eta$ vanishes with $r$. For a meridian curve of the equipotential surface $V=$ const., we find

$$
y= \pm e^{\mu}\left(2 x+e^{\mu}\right)^{1 / 2} .
$$

These meridian curves, for $V>1$, enclose the loaded segment, except near the origin, 0 , where they have contact with it of infinite order.

The equipotential surface $V=2$, together with the point 0 , bounds an infinite domain, $T$. The Dirichlet problem for $T$ and the boundary value 2 is impossible. The function $V$, which fulfills every other requirement, fails to approach 2 at 0 . In fact at this point $V$ approaches 1 along every ray terminating at 0 other than the loaded segment.

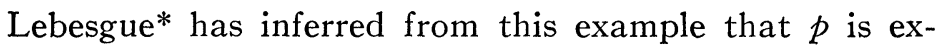
ceptional for a domain $T$ if all the boundary near $p$ lies inside a surface of revolution whose meridian curve is of the form $y=e^{-A / x},(x>0, A>0), p$ being at the vertex.

On the other hand, by means of superharmonic barriers of the form $U=\left(x^{\alpha}+y^{\alpha}-z\right)^{1 / 2}, \alpha=2 /(2 n+1)$, he shows that $p$ is regular if it is the vertex of a surface of revolution of the form $z=A r^{\beta},\left(A>0,0<\beta<1, r=\left(x^{2}+y^{2}\right)^{1 / 2}\right)$, which lies outside of $T$ in a neighborhood of $p$, except at $p$ itself. The results are extended to the case of certain spines which are not surfaces of revolution.

* Comptes Rendus, vol. 178 (1924), p. 349. 
Bouligand* has constructed barriers for regions with a general type of reentrant conical point as follows. $U=$ $r^{\alpha} \psi(\phi, \theta)$ is harmonic in the interior of any cone in which $\psi(\phi, \theta)$ is a continuous differentiable solution of the equation $\Delta_{2} \psi+\alpha(\alpha+1) \psi=0$, where $\Delta_{2} \psi$ is the second differential parameter of Beltrami for the surface of the unit sphere. Let $S$ denote a simply connected domain of this sphere with more than one boundary point. Green's function, $g(P, Q)$, exists for $S$ and the differential equation $\Delta_{2} g=0$, and any solution of the differential equation for $\psi$ which vanishes everywhere on the boundary of $S$ satisfies the integral equation

$$
\psi(P)=\frac{\alpha(\alpha+1)}{2 \pi} \iint_{S} g(P, Q) \psi(Q) d S_{Q},
$$

and conversely. There exists a solution $\psi_{0}(\phi, \theta)$, of this equation, positive in $S$, approaching 0 on the boundary, and corresponding to a positive characteristic number, $\lambda_{0}$. Accordingly, if $\alpha$ is the positive root of $\alpha(\alpha+1)=2 \pi \lambda_{0}$, $U(P)=r^{\alpha} \psi_{0}(\phi, \theta)$ is a barrier for the origin for any domain which admits as strictly exterior (save at the origin), as much of the cone subtended at the origin by the complement on the unit sphere of $S$ as lies in some neighborhood of the origin. For $U$ will be harmonic in $T$ in a neighborhood of the origin, and will approach 0 there but at no other boundary point of $T$ in this neighborhood.

In particular, the domain $S$ may consist of the whole surface of the unit sphere save for an arc of a great circle, or other curve, so that $p$ is regular for $T$ if it is the vertex of a triangle, either flat, or conical and bounded by two elements and a third geodesic, which lies outside $T$ except at $p$.

Raynor $\dagger$ obtained the following condition. The point $p$ is regular for $T$ provided there is a number $\eta>0$, such

* Application de la notion du prolongement des fonctionnelles à l'étude de l'existence de la solution du problème de Dirichlet harmonique, Bulletro des Sciences Mathématiques, (2), vol. 48 (1924), pp. 1-19.

$\dagger$ Dirichlet's problem, Annals of Mathematics, (2), vol. 23 (1923), pp. 183-197. 
that on an infinite number of spheres about $p$ with radii approaching 0 , the ratio of the Lebesgue measure of the set of points of each sphere exterior to $T$ to the whole area of that sphere, is greater than $\eta$.

Bouligand* has given special attention to the character of the infinite point as a boundary point. It is not difficult to show that an inversion which carries a finite boundary point into a finite point does not alter the regularity or exceptional character of this boundary point. Accordingly, it is natural to define the infinite point as regular or exceptional according to the character of the point into which it is transformed by an inversion. If $p$ is a finite exceptional boundary point for a domain $T$, there exists a sequence of points of $T,\left[P_{k}\right]$ with $p$ as limit, such that $G\left(P_{k}, Q\right) \rightarrow \eta>0$, as $P \rightarrow p$. Accordingly, $u_{p}(P)=\lim G\left(P, P_{k}\right)$ is a never negative, not identically vanishing function, harmonic in $T$, approaching 0 at all regular boundary points, and dominated by $1 / P p$. An inversion in $p$ leads to this result: a necessary condition that the infinite point be exceptional for a domain $T^{\prime}$ is the existence of a function positive, bounded, and harmonic in $T^{\prime}$ and vanishing at all finite regular boundary points. The converse can also be proved, provided the exceptional points of $T^{\prime}$ are not too numerous. From this Bouligand also inferred independently the result of Raynor.

The above particular criteria for the character of boundary points have been given in some detail to illustrate methods of investigation. It should be added, however, that Wiener $\dagger$ has shown how they may be derived from a general criterion of his in terms of the capacity of point sets, a topic to which we devote the next section. We close the present section with a remarkable result of Bouligand's : If $p$ is an ex-

* Sur les principes de la théorie du potentiel, Bulletin DES SCIEnCes Mathematiques, (2), vol. 48 (1924), pp. 1-12.

$\dagger$ The Dirichlet problem, Journal of Mathematics and Physics, Mass. Inst. Tech., vol. 3 (1924), pp. 127-146.

‡ Loc. cit., Annales de la Société Polonaise. 
ceptional point of $T$, and if $U(P)$ is the sequence solution of the Dirichlet problem corresponding to boundary values which are 0 in the neighborhood of $p$, and nowhere negative, then the arithmetic mean of the values of $U(P)$ on a sphere about $p$ approaches, as the radius approaches 0 , the upper limit of $U(P)$ at $p$. As the limit of the ratio of the measure of the set of points outside $T$ and on the sphere to the whole area is 0 as the radius approaches 0 , the fact that $U(P)$ may not be defined at all points of the spheres causes no difficulty in interpreting this arithmetic mean.

7. Capacity. The electrostatic capacity of a conductor may be defined as the charge necessary to raise it to unit potential, when isolated. The concept may be applied to any bounded point set (and we need it for no others) as follows. Let $B$ be such a set, and $B^{\prime}$ the set obtained by adjoining to $B$ its limit points. Now $B^{\prime}$ may bound various domains, but $B^{\prime}$ will certainly contain the complete boundary $t$, of a domain $T$, extending to infinity. The sequence solution of the Dirichlet problem for $T$ and the boundary values 1 on $t$ (and vanishing at infinity) is called the conductor potential of the set $B$. If $v(P)$ denote this conductor potential, Gauss' integral,

$$
\frac{1}{4 \pi} \iint \frac{\partial v}{\partial n} d S
$$

taken over any smooth simple surface containing $B$ in its interior, gives the capacity of $B$. A number of properties of the capacities of sets have been derived by Wiener, Bouligand, and the author.* But the outstanding service which the concept has rendered lies in Wiener's necessary and sufficient condition for the regularity of a boundary point: Let $p$ denote a boundary point of $T$, and let $\gamma_{n}$ denote the capacity of the set of points not in $T$ whose distances from $p$ lie in the closed interval $\left(\lambda^{n+1}\right.$, $\lambda^{n}$ ), where $\lambda$ is a number between 0 and 1 . Then $p$ is re-

* Loc. cit., I. 
gular or exceptional according as the series $\sum_{1}^{\infty}\left(\gamma_{n} / \lambda^{n}\right)$ diverges or converges.

Wiener has given the corresponding theorem for 2 and $n>3$ dimensions.

A proof of the above theorem, somewhat modified, and free from the use of Stieltjes integrals, follows. We first assume the series $\sum_{1}^{\infty}\left(\gamma_{n} / \lambda^{n}\right)$ divergent, and show how it follows that a barrier for $T$ at $p$ can be constructed. From our assumptions, it follows that one of the three series $\sum_{1}^{\infty}\left(\gamma_{3 n} / \lambda^{3 n}\right), \sum_{1}^{\infty}\left(\gamma_{3 n+1} / \lambda^{3 n+1}\right), \sum_{1}^{\infty}\left(\gamma_{3 n+2} / \lambda^{3 n+2}\right)$, must diverge. To fix ideas, suppose it is the first-the notation, not the reasoning, would have to be altered to meet the other two cases. Let $B_{n}$ denote the point set of the theorem, whose capacity is $\gamma_{n}$, and let $V_{m}(P)$ denote the conductor potential of the set containing all the points of $B_{3 m}, B_{3 m+3}, B_{3 m+6}, \cdots$. The heart of the argument is to show that $V_{m}(P) \rightarrow 1$ as $P \rightarrow p$.

This is done by building up a sequence of harmonic functions which are dominated by $V_{m}(P)$. The first is harmonic in the region within the sphere $\sigma_{3 m-1}$, with center at $p$ and radius $\lambda^{3 m-1}$, except for the set $B_{3 m}$ (and also except for any domains which $B_{3 m}$ may completely bound, if there are any). It is the sequence solution, $v_{1}(P)$, corresponding to values 0 on $\sigma_{3 m-1}$ and 1 on $B_{3 m}$. Then we have $V_{m}(P) \geqq v_{1}(P)$ at the points at which both are harmonic. We seek a lower bound for $v_{1}(P)$ on $\sigma_{3 m+2}$.

By definition, $v_{1}(P)$ is the limit of a sequence of functions $\left[u_{n}(P)\right], u_{n}(P)$ being harmonic in a normal region $T_{n}$, bounded by $\sigma_{3 m-1}$ and by a smooth surface, $t_{n}$, interior to $\sigma_{3 m-1}$ and enclosing $B_{3 m}$, the domains $T_{n}$ having the domain of $v_{1}(P)$ as limit, and the boundary values of $u_{n}(P)$ being 0 on $\sigma_{3 m-1}$ and 1 elsewhere. Then

$$
u_{n}(P)=\frac{1}{4 \pi} \iint\left(G \frac{\partial u_{n}}{\partial n}-u_{n} \frac{\partial G}{\partial n}\right) d S_{Q},
$$

where $G$ is Green's function for $\sigma_{3 m-1}$, and the integral is taken over the boundary of $T_{n}$; or, since $u_{n}$ and $G$ vanish 
on $\sigma_{3 m-1}$, over $t_{n}$. The positive sense of the normal $n$ is the outward one. Accordingly,

$$
u_{n}(P)=\frac{\bar{G}}{4 \pi} \iint \frac{\partial u_{n}}{\partial n} d S_{Q},
$$

where $\bar{G}$ is a mean value of $G$. Now $\partial u_{n} / \partial n$ is never less than the corresponding derivative of the conductor potential of $t_{n}$, and hence* than that of $B_{3 m}$, and therefore $u_{n}(P) \geqq \bar{G} \gamma_{3 m}$. We conclude that in the limit $v_{1}(P) \geqq \min G \gamma_{3 m}$, where $P$ is on $\sigma_{3 m+2}$, and $Q$ on $B_{3 m}$, i. e., $p P=\lambda^{3 m+2}$ and $p Q=$ $\leqq \lambda^{3 m}$. We find for the minimum of Green's function for a sphere of radius $c$, when the distances from the center of $P$ and $Q$ do not exceed $a$ and $b$, respectively, the value

$$
\frac{1}{a+b}-\frac{c}{c^{2}+a b}
$$

or, in our present case,

$$
\min G \geqq \frac{1}{\lambda^{3} m} \frac{(1-\lambda)\left(1-\lambda^{3}\right)}{\left(1+\lambda^{2}\right)\left(1+\lambda^{4}\right)}=\frac{\alpha}{\lambda^{3 m}},
$$

where $\alpha$ is independent of $m$. Hence, for $P$ on or within $\sigma_{3 m+2}, v_{1}(P) \geqq \alpha \gamma_{3 m} / \lambda^{3 m}$.

Next, consider the sequence solution $v_{2}(P)$ corresponding to the points within the same sphere, $\sigma_{3 m-1}$, except for the two sets, $B_{3 m}$ and $B_{3 m+3}$, and corresponding to boundary values 0 on $\sigma_{3 m-1}$, and 1 elsewhere. This function again is dominated by $V_{m}(P)$, and it dominates $v_{1}(P)$. Hence $v_{2}(P)-\alpha \gamma_{3 m} / \lambda^{3 m} \geqq 0$ on $\sigma_{3 m+2}$ and corresponds to values $1-\alpha \gamma_{3 m} / \lambda^{3 m}$ on $B_{3 m+3}$, and so, on $\sigma_{3 m+5}$, is not less than $1-\alpha \gamma_{3 m} / \lambda^{3 m}$ times the function corresponding to $v_{1}(P)$ with $3 m$ replaced by $3 m+3$. That is, on and within $\sigma_{3 m+5}$,

$$
v_{2}(P)-\alpha \gamma_{3 m} / \lambda^{3 m} \geqq\left(1-\alpha \gamma_{3 m} / \lambda^{3 m}\right) \alpha \gamma_{3 m+3} / \lambda^{3 m+3},
$$

or

$$
v_{2}(P) \geqq 1-\left(1-\alpha \gamma_{3 m} / \lambda^{3 m}\right)\left(1-\alpha \gamma_{3 m+3} / \lambda^{3 m+3}\right) .
$$

* Kellogg, loc. cit., I. 
Proceeding in this way, with $v_{r}(P)$ the sequence solution corresponding to values 0 on $\sigma_{3 m-1}$ and 1 on $B_{3 m}, B_{3 m+3}, \cdots$, $B_{3(m+r-1)}$, we find that within $\sigma_{3(m+r)-1}$,

$$
V_{m}(P) \geqq V_{r}(P) \geqq 1-\prod_{i=0}^{r-1}\left(1-\alpha \gamma_{3(m+i)} / \lambda^{3(m+i)}\right) .
$$

But since, by hypothesis, the infinite series $\sum_{1}^{\infty}\left(\gamma_{3 n} / \lambda^{3 n}\right)$ diverges, the infinite product $\Pi_{1}^{\infty}\left(1-\alpha \gamma_{3 n} / \lambda^{3 n}\right)$ diverges to 0 , and hence there is a sphere about $p$ within which $V_{m}(P)$ is arbitrarily close to 1 .

This property of $V_{m}(P)$ being established, it is seen that

$$
U_{p}(P)=\sum_{1}^{\infty} \frac{1}{2^{m}}\left(1-V_{m}(P)\right)
$$

is a barrier for $T$ at $p$, for the series is in $T$ a uniformly convergent series of harmonic functions approaching 0 at $p$, and elsewhere positive, with positive boundary values.

Suppose now that $\sum_{1}^{\infty}\left(\gamma_{n} / \lambda^{n}\right)$ converges. We shall see that the assumption that $p$ is regular leads to a contradiction. Let $m$ be chosen so that $\sum_{m}^{\infty}\left(\gamma_{n} / \lambda^{n}\right)<\lambda / 4$. Then, if $p$ is regular, the conductor potential, $V_{m}(P)$, of the points within $\sigma_{m}$ but not in $T$, approaches 1 at $p$, since the sequence solution approaches the assigned values at regular boundary points. Hence there is a sphere, $\sigma^{\prime}$, about $p$, within which $V_{m}(P)>3 / 4$. On the other hand, for sufficiently large $k$, $V_{m+k}(P)$, being the conductor potential of a set within $\sigma_{m+k}$, cannot exceed $1 / 4$ on $\sigma^{\prime}$. Now the conductor potential of the sum of two point sets never exceeds the sum of the conductor potentials. ${ }^{*}$ Hence, if $V_{m, m+k}$ denotes the conductor potential of the set of points not in $T$ and whose distances from $p$ lie in the closed interval $\left(\lambda^{m+k+1}, \lambda^{m}\right)$, $V_{m} \leqq V_{m+k}+V_{m, m+k}$, and hence, in particular, on $\sigma^{\prime}$, $3 / 4<1 / 4+V_{m, m+k}$, that is $V_{m, m+k}>1 / 2$.

On the other hand, $V_{m, m+k} \leqq \sum_{m}^{m+k} v_{n}$, where $v_{n}$ is the conductor potential of $B_{n}$. But the value of a conductor

* Kellogg, loc. cit., I. 
potential is never decreased at an external point, $p$, by moving the whole charge to the point of the set nearest $p$, and hence $V_{m, m+k} \leqq \sum_{m}^{m+k}\left(\gamma_{n} / \lambda^{n+1}\right)<1 / 4$. Thus a contradiction has been arrived at, and $p$ must be exceptional.

8. Removable Singularities. Another problem which is illuminated by the notion of capacity is that of the removable singularities of bounded harmonic functions. We have seen that in the plane, isolated points, and in space of three dimensions, arcs of curves, can be the seats of no singularities of bounded harmonic functions other than removable ones. The following is the complete generalization of these facts.

Let $T$ denote a domain, and let $B$ denote a sub-set of its boundary with the properties $a$ ) the set $T^{\prime}=T+B$ is a domain (open continuum), and $b$ ) $B$ has capacity 0 . Then any function, bounded and harmonic in $T$, may be so defined on $B$ as to be harmonic in $T^{\prime}$. Conversely, if $B$ is a set with the property $a$, and if no bounded harmonic function in $T$ can have singularities at the points of $B$ other than removable ones, then $B$ must have 0 capacity.*

There exists also a certain maximal set $B$, such that no further boundary points can be added to it without impairing one of the above properties. This is the complete improper set of Bouligand, consisting of all points at which $\lim G(P, Q)>0$.

We have stated (p. 611) that a regular boundary point of a plane domain must be a point of condensation of the boundary, and the assertion is based upon the fact that a closed denumerable set has capacity 0 . This is included in a theorem of Bouligand: $\dagger$ A necessary and sufficient condition that $B$ have capacity 0 is that given any distribution of positive masses on $B$ such that any sphere containing points of $B$ contains a positive total mass, the potential of this distribution shall have the upper limit $+\infty$ at each point of $B$.

* Kellogg, loc. cit., I.

$\dagger$ Annales de la Société Polonaise, loc. cit. p. 103. 
For if to each point, $P_{n}$, of the denumerable set we assign a mass $1 / n^{2}$, the potential will have the required property.

9. Uniqueness. If at any boundary point a harmonic function fails to approach a boundary value continuously, then boundary values no longer determine it uniquely, as Schwarz* first observed. The situation whose existence he pointed out is present in the case of every domain which is not normal. For, as we have seen, there exist functions, positive in such a domain, and approaching 0 at every regular boundary point (p. 615). But these functions may not be bounded. In many cases, the additional requirement that the function be bounded is sufficient to insure uniqueness. The question of the unique determination of harmonic functions by continuous boundary values for non-normal domains has not yet attained a satisfactory solution. The following is a partial result in this direction.

If the exceptional points of a domain $T$ with bounded boundary form a set of capacity 0 , then there is one and only one function, bounded and harmonic in $T$, which assumes preassigned continuous values at every regular boundary point. This is obvious, since the difference of two such functions would be dominated by a constant times the conductor potential of any set enclosing the exceptional points of the boundary of $T$, i. e., of a set of arbitrarily small capacity. Such a conductor potential, however, can be made arbitrarily small at any given point of $T$ by sufficiently shrinking up the set of which it is the conductor potential. For domains of this character, then, the sequence solution comes as near to solving the Dirichlet problem as any bounded function can.

10. Discontinuous Boundary Values. We are primarily concerned in this review with the Dirichlet problem for continuous boundary values. But generalizations in the direction of discontinuous boundary values have been attained which are too significant not to receive attention.

* Gesammelte Werke, vol. 2, p. 194. 
The investigations in question started with simple domains. Studying the circle, Fatou* showed that if $f(\theta)$ is periodic, bounded and summable, Poisson's integral formed with $f(\theta)$ approaches $f(\theta)$ along radii at all points of the circumference with possible exception of a set of measure 0 . Moreover, no other bounded harmonic function approaches the same boundary values in the same way. These results were extended by the author $\dagger$ to domains of finite connectivity the coordinates of whose boundary as functions of the arc length have second derivatives satisfying uniformly a generalized Lipschitz condition; it was shown also that for approach along a radius may be substituted approach along any curve meeting the boundary orthogonally with finite curvature.

Evans $\ddagger$ carried the generalization to any domain whose boundary consists of a finite number of connected sets. If $h(P, Q)$ is a conjugate of Green's function, there is, for fixed $Q$, a one to one correspondence between the accessible boundary points and the values of $h$ between 0 and $2 \pi$, with exceptions which do not affect the Lebesgue integral. If the boundary values constitute a bounded summable function of $h$ (and if they do so for one position of the pole, $Q$, they will for all positions), then there is one and only one function, bounded and harmonic in $T$ with first partial derivatives which are summable in $T$, which takes on the given values on the boundary for all values of $h$ except for a set of measure 0 . This function is given by Green's integral.

* Séries trigonométriques et séries de Taylor, Acta Mathematica, vol. 30 (1906), p. 339 et seq.

$\dagger$ Harmonic functions and Green's integral, TRANSACTIONS OF THIS SocIETY, vol. 13 (1912), pp. 109-132.

$\ddagger$ Fundamental points of potential theory, Rice Institute Pamphlet, vol. 7 (1920), pp. 252-329, especially p. 327; Problems of potential theory, Proceedings of the National Academy, vol. 7 (1921), pp. 89-98; The Dirichlet problem for the general finitely connected region, PROCEEDINGS oF THE Toronto Congress. 
Evans and Bray* have studied Poisson's integral and Green's integral for plane domains of finite connectivity when expressed as Stieltjes integrals with respect to the boundary values, obtaining necessary and sufficient conditions on a harmonic function that it be so expressible. They have announced similar results for the sphere. $\dagger$

The Dirichlet problem for a general domain and for any bounded boundary values has been treated by Perron $\ddagger$ and Remak§ in essentially the following way. Let $\bar{F}(p)$ and $F(P)$ be the upper and lower limit functions of the assigned boundary values, $F(p)$. A function is called an upper function for these boundary values if it is continuous in $T+t$ and superharmonic, and is not less than $\bar{F}(p)$ on the boundary, $t$. It is a lower function if continuous in $T+t$ and subharmonic and not greater than $\underline{F}(p)$ on $t$. The lower limit, $u$, of the set of all upper functions for $F(p)$, and the upper limit, $v$, of the set of all lower functions for $F(p)$ are harmonic in $T$. By means of barriers, it is shown that if $T$ satisfies a condition employed by Lebesgue (the conclusion holds if $T$ is any normal domain), $\underline{u}(p) \geqq \underline{F}(p)$ and $\bar{u}(p) \leqq \bar{F}(p)$. Hence, in particular, if $T$ is normal and $F(p)$ continuous, $u$ is the solution of the Dirichlet problem.

Furthermore, whether $T$ is normal or not, $F(p)$ being continuous, $u$ and $v$ coincide, and are identical with the

* Sur l'intégral de Poisson généralisée, Comptes Rendus, vol. 176 (1923), p. 1042; La formule de Poisson et le problème de Dirichlet, Comptes Rendus, vol. 176 (1923), p. 1368; also Evans, Sur l'intégral de Poisson, Comptes Rendus, vol. 177 (1923), p. 241; Evans, The Dirichlet problem for the general finitely connected region, ProceEdings of the TORONTO CONGRESS.

$\dagger$ This Bulletin, vol. 29 (1923), p. 210.

$\ddagger$ Eine neue Behandlung der ersten Randwertaufgabe für $\Delta u=0$, MATHEmatische Zeitschrift, vol. 18 (1923), pp. 42-54. See also Rad6 and F. Riesz, Über die erste Randwertaufgabe für $\Delta u=0$, Mathematische ZeiT ${ }^{-}$ SCHRIFT, vol. 22 (1925), pp. 41-44.

$\S \ddot{U b e r}$ potentialkonvexe Funktionen, Mathematische Zeitschrift, vol. 20 (1924), pp. 126-130.

If Loc. cit., Rendiconti di Palermo. 
sequence solution.* Thus the sequence solution appears stripped of the unessential elements involved in the nested set of domains and the continuous extension to $T$ of the boundary values.

In very simple cases, however, $u$ and $v$ need not coincide; for instance, in the case of the circle for boundary values which are 1 at points corresponding to rational values of the angular coordinate, and 0 elsewhere. Wiener $\dagger$ has considerably extended the class of boundary functions to which a harmonic function can be assigned, in a paper in which a harmonic function is regarded as a homogeneous linear functional of its boundary values. The analytic expression employed for the harmonic function is a form of generalized integral due to Daniell. $\ddagger$ Broad conditions for the existence of this integral are set up in terms of capacity. If it exists, the harmonic function it represents has upper and lower limits at a boundary point, $p$, which lie in the closed interval determined by the corresponding limits of the boundary function.

11. Conclusion. From the above account, it should appear that the Dirichlet problem has recently been through a period of remarkable development. But it is characteristic of scientific progress that each advance raises new questions. It may not be out of place to indicate here some connected with the Dirichlet problem.

With respect to general domains, there is the question of the frequency of possible exceptional points. Can they form a set of positive capacity? Must every sub-set of a boundary contain regular points, if the sub-set is closed and has positive capacity? Are there domains admitting

* Wiener, Note on a paper of $O$. Perron, Journal of Mathematics AND Physics, Mass. Inst. of Tech., vol. 4 (1925), pp. 21-32.

$\dagger$ Discontinuous boundary conditions and the Dirichlet problem, TransACtions of this Society, vol. 23 (1925), pp. 307-314.

$\ddagger$ g general form of integral, Annals of Mathematics, (2), vol. 19 (1917-18), pp. 279-294. 
a bounded harmonic function other than 0 which approaches 0 at every regular boundary point?

Further questions arise in the study of boundary values which may be different for the different possible sets of curves of approach to a multiple boundary point, yet which are continuous in the sense that the difference in the boundary values at $p$ and $q$ approaches 0 with the lower limit of the length of the curves lying in $T$ except for their end points, and connecting $p$ and $q$ (these points being accessible). It should not be difficult to study the existence and properties of the sequence solution in this case. To be sure, Evans' results hold for such boundary values. But the domains he has treated so far are normal ones save for possible isolated boundary points.

There are also interesting problems connected with capacity. Particularly needed are further inequalities connecting the capacity of a set with the capacities of its subsets. Is it possible to express conditions for regularity in some even more purely geometric form than those in terms of capacity?*

For the most part, recent investigations have been concerned with non-overlapping domains. $\dagger$ Generalizations in this respect should be considered. Finally, a number of questions suggest themselves in connection with the theory of functions of a complex variable, and the extension of the results gained for Laplace's equation to other differential equations of elliptic type has scarcely begun. $\neq$

HARVARD UNIVERSITY

* In this connection, see the interesting note of Bouligand, Dimension, étendu, et densité, Comptes Rendus, vol. 180 (1925), pp. 246-48.

$\dagger$ But Perron (loc. cit.) admits overlapping, provided the domain contains no branch points. See also Hurwitz-Courant, Funktionentheorie, 2d ed., Berlin, 1925.

$\ddagger$ The reader desiring references to the literature besides those given above, will find an excellent bibliography in Bouligand's Fonctions harmoniquès. Principes de Picard et de Dirichlet, Mémorial des Sciences Mathématrques, Paris, Gauthier-Villars, 1926. 